\title{
Spherically symmetric solution with Dirac scalar field in Cartan-Weyl space
}

\section{Babourova O.V., Frolov B.N., Romanova E.V.}

Moscow State Pedagogical University, Moscow, Russia;

E-mail: Babourova <baburova@orc.ru>,Frolov<frolovbn@orc.ru>,Romanova <solntce_07@mail.ru>;

On the basis of the modified variational principle a spherically symmetric solution of the theory of gravitation with the Weyl-Dirac scalar in Cartan-Weyl space is found. The metrics of this solution coincides with the Yilmaz-Rosen metric, and the solution for the Weyl-Dirac scalar field is $\beta_{\infty} \exp ( \pm k / r)$. The hypothesis that the Weyl-Dirac scalar field represents the main component of dark matter is stated.

Keywords: variational principle, Cartan-Weyl space, Weyl-Dirac scalar field, spherically symmetric solution .

DOI: $10.18698 / 2309-7604-2015-1-49-59$

\section{Introduction}

Now at construction of the modern theory of gravitation three types of post-Riemannian spaces are used. This is a general affine-metric space characterized by curvature $\mathrm{R}^{a}{ }_{b}$ and torsion $\mathrm{T}^{a}$ 2-forms, and also by a nonmetricity $\mathrm{Q}_{a b}$ 1-form,

$$
R_{b}^{a}=d \Gamma_{b}^{a}+\Gamma_{c}^{a} \wedge \Gamma_{b}^{c}, T^{a}=d \theta^{a}+\Gamma_{b}^{a} \wedge \theta^{b}, Q_{a b}=-\left(\nabla_{\mu} g_{a b}\right) h_{c}^{\mu} \theta^{c}
$$

where $\theta^{a}$ - cobasis 1 -forms, $h_{a}^{\mu}$ - tetrad coefficients, $g_{a b}$-components of tangent space metric tensor, $\Gamma_{b}^{a}-$ a post-Riemannian space connection 1-form, $\wedge-$ a symbol of external multiplication, $\mathrm{d}-$ an external differentiation operator, $\nabla_{\mu}-$ a covariant post-Riemannian differentiation symbol.

Other type of spaces is a Cartan-Weyl space $C W_{4}$, which is a special case of the general affine-metric space, if the nonmetricity 1-form submits to a Weyl condition,

$$
Q_{a b}=\frac{1}{4} g_{a b} Q, Q=Q_{c} \theta^{c}, Q=g^{a b} Q_{a b}, Q_{c}=g^{a b} Q_{a b c}=Q_{a c}^{a}
$$


Here $\mathrm{Q}$ is a Weyl 1-form Вейля, and $Q_{a}$ is a Weyl vector.

At last, the third one is a Riemann-Cartan space $R C_{4}$, which is a special case of a CartanWeyl space, in which the Weyl vector is equal to zero, $Q_{a}=0$.

In [1]-[3] a gauge theory of Poincaré-Weyl group was constructed, in which the Poincaré group was added with a group of spacetime expansions and compressions (dilatations). In mathematical sense the group of dilatations is equivalent to the Weyl's group of scale transformations. As a consequence of this theory, spacetime appears to be allocated of CartanWeyl space geometrical properties, and such orientation of basis can be chosen in its tangent spaces that components of tangent space metric tensor can be chosen in the form,

$$
g_{a b}=\beta^{2}(x) g_{a b}^{M}
$$

where $g_{a b}^{M}$ are constant components of Minkowski metrics, and an arbitrary function of spacetime points $\beta(x)$ describes some scalar field of geometrical nature.

The given statement represents a special case of the following statement, proved in the monograph [4] for the general affine-metric space $L_{4}(g, \Gamma)$.

Lemma (B.N.Frolov, 2003). A tangent space basis of the general affine-metric space can not be chosen in a gauge covariant form as a "rigid" basis, in which all metric tensor components represent a set of the same numbers in each spacetime point.

On the basis of the specified results, in [5]-[11] the conformal theory of gravitation in a CartanWeyl space was advanced. Application of the given theory for super-early evolution of the universe has allowed to receive an exponential reduction in due course an effective cosmological constant $\Lambda \beta^{4}$ that has in turn allowed to give a way of overcoming a well-known "cosmological constant problem".

In [12] the new variational principle in a Cartan-Weyl space was formulated, which modified a variational principle in this space, advanced in [5]-[11], [13]. This new variational principle has several advantages in comparison with accepted earlier. 


\section{The modified variational principle in a Cartan-Weyl space with scalar Weyl-Dirac field}

According to the gauge gravitational theory of Poincaré-Weyl group [1]-[3], spacetime has Cartan-Weyl geometrical structure, and besides there should be an additional structure as geometrical scalar field $\beta$.

The physical substantiation of advantage of the Poincaré-Weyl group as a group of spacetime invariant properties is based on the fact that the high energy physics finds out property of a scale invariance (Bjorken scaling, the 1990 Nobel Prize in physics), becomes as well apparent in cosmology. At an early stage of the universe evolution, when rest masses of elementary particles did not arise yet, all interactions were carried out by massless quanta. In this case these interactions have property of a scale invariance (independence of absolute size of intervals), and the symmetry group of space, in which these fields exist, is the Poincaré-Weyl group.

So, a hypothesis about scale invariance, according to which an amplitude of initial fluctuations of density were identical in all scales, underlies calculation of an initial part of a spectrum of initial fluctuations of density of a matter in the early universe (Harisson-Zel'dovich plateau), confirmed with COBE experiment with studying anisotropy of brightness of relic radiation (the 2006 Nobel Prize in physics).

In [5]-[7] in a tetrad formalism and in [8]-[11] in a formalism of external forms, the variational principle for the conformal theory of gravitation in a Cartan-Weyl space with additional structure as a geometrical scalar field was advanced. At this theory the scalar field had the properties similar to a scalar field, entered by Dirac in the well-known work [14], and earlier by Deser [15]. This field was named as Dirac scalar field.

In [12] the modified variational formalism is advanced, according to which the scalar field $\beta$ is not entered in the theory by "hands" irrespective of the metric (how it was carried out in [5] -[11]), but this scalar field is entered in a Lagrangian density as a representation of a tangent space metric as (1.2) with the help of a method of Lagrange uncertain multipliers. In this case properties of this scalar field are substantially determined by ideas of the old Weyl's gauge theory [16], and this field is expedient for naming as Weyl-Dirac scalar field.

The Lagrangian density 4-form of the theory we shall present as

$$
L=L_{G}+\Lambda^{a b} \wedge\left(Q_{a b}-(1 / 4) g_{a b} Q\right)+\lambda^{a b}\left(g_{a b}-\beta^{2} g_{a b}^{M}\right) \eta
$$




$$
\begin{aligned}
& \mathrm{L}_{G}=2 f_{0}\left[(1 / 2) \mathrm{R}^{a}{ }_{b} \wedge \eta_{a}{ }^{b}+\right. \\
& +\rho_{1} \mathrm{~T}^{a} \wedge * \mathrm{~T}+\rho_{2}\left(\mathrm{~T}^{a} \wedge \theta_{b}\right) \wedge *\left(\mathrm{~T}^{b} \wedge \theta_{a}\right)+\rho_{3}\left(\mathrm{~T}^{a} \wedge \theta_{a}\right) \wedge *\left(\mathrm{~T}^{b} \wedge \theta_{b}\right)+ \\
& \left.+16 \xi \mathrm{Q}_{a b} \wedge * \mathrm{Q}^{a b}+4 \zeta \mathrm{Q}_{a b} \wedge \theta^{a} \wedge * \mathrm{~T}^{b}+l \mathrm{~d} \ln \beta \wedge * \mathrm{~d} \ln \beta\right] .
\end{aligned}
$$

Here $\eta_{a}^{b}=*\left(\theta_{a} \wedge \theta^{b}\right) ; f_{0}=c^{4} / 16 \pi G, \rho_{1}, \rho_{2}, \rho_{3}, \xi, \zeta, I$ are coupling constants; $\Lambda^{a b}$ and $\lambda^{a b}$ are Lagrange uncertain multipliers, and we have as consequence of the Weyl condition (1.1), $g_{a b} \Lambda^{a b}=0$. In comparison with [5]-[11] here the term with cosmological constant is omitted as the local task will consider, and also a Lagrange density of external fields is omitted as the problem in emptiness will be solved further. Last term in (2.2) is added for maintenance of dynamics of the scalar field $\beta$.

In order to derive the equations of a gravitational field in emptiness it is necessary to vary (3.1) and (3.2) independently with respect to the basic forms $\theta^{a}$ ( $\theta$-equation), to the connection 1-form $\Gamma_{b}^{a}$ ( $\Gamma$-equation), to the components of the tangent space metric $g_{a b}$ ( $g$-equation), to the field $\beta$ and to the Lagrange multipliers using a Lemma about result of commuting of variation operator and Hodge dual conjugation [17].

In a result the $\Gamma$-equation reads,

$$
\begin{aligned}
& f_{0}\left[-\frac{1}{4} \mathcal{Q} \wedge \eta_{a}{ }^{b}+\frac{1}{2} \mathcal{T}_{c} \wedge \eta_{a}^{b c}+\frac{1}{2} \eta_{a c} \wedge \mathcal{Q}^{b c}+2 \rho_{1} \theta^{b} \wedge * \mathcal{T}_{a}+\right. \\
& +2 \rho_{2} \theta^{b} \wedge \theta_{c} \wedge *\left(\mathcal{T}^{c} \wedge \theta_{a}\right)+2 \rho_{3} \theta^{b} \wedge \theta_{a} \wedge *\left(\mathcal{T}^{c} \wedge \theta_{c}\right)+ \\
& \left.+4 \xi \delta_{a}^{b} *+\xi\left(2 \delta_{a}^{b} \theta^{c} \wedge \mathcal{T}_{c}+\theta^{b} \wedge *\left(\mathcal{Q} \wedge \theta_{a}\right)\right)\right]-\Lambda_{a}^{b}=0,
\end{aligned}
$$

the $\boldsymbol{\theta}$-equation reads, 


$$
\begin{aligned}
& \frac{1}{2} \mathcal{R}_{c}^{b} \wedge \eta_{b a}^{c}+ \\
& +\rho_{1}\left[2 \mathrm{D} * \mathcal{T}_{a}+\mathcal{T}_{b} \wedge *\left(\mathcal{T}^{b} \wedge \theta_{a}\right)+*\left(* \mathcal{T}^{b} \wedge \theta_{a}\right) \wedge * \mathcal{T}_{b}\right]+ \\
& +\rho_{2}\left[2 \mathrm{D}\left(\theta_{b} \wedge *\left(\mathcal{T}^{b} \wedge \theta_{a}\right)\right)+2 \mathcal{T}^{b} \wedge *\left(\theta_{b} \wedge \mathcal{T}_{a}\right)-\right. \\
& \left.-*\left(\mathcal{T}^{b} \wedge \theta_{c} \wedge \theta_{a}\right)\left(\mathcal{T}^{c} \wedge \theta_{b}\right)-*\left(*\left(\mathcal{T}^{c} \wedge \theta_{d}\right) \wedge \theta_{a}\right) \wedge *\left(\mathcal{T}^{d} \wedge \theta_{c}\right)\right]+ \\
& +\rho_{3}\left[2 \mathrm{D}\left(\theta_{a} \wedge *\left(\mathcal{T}^{b} \wedge \theta_{b}\right)\right)+2 \mathcal{T}_{a} \wedge *\left(\mathcal{T}^{b} \wedge \theta_{b}\right)-\right. \\
& \left.-*\left(\mathcal{T}^{b} \wedge \theta_{b} \wedge \theta_{a}\right)\left(\mathcal{T}^{c} \wedge \theta_{c}\right)-*\left(*\left(\mathcal{T}^{b} \wedge \theta_{b}\right) \wedge \theta_{a}\right) \wedge *\left(\mathcal{T}^{c} \wedge \theta_{c}\right)\right]+ \\
& +\xi\left[-\mathcal{Q} \wedge *\left(\mathcal{Q} \wedge \theta_{a}\right)-*\left(* \mathcal{Q} \wedge \theta_{a}\right) * \mathcal{Q}\right]+\zeta\left[\mathrm{D} *\left(\mathcal{Q} \wedge \theta_{a}\right)-\right. \\
& \left.\left.+I\left[-d \ln \beta \wedge \theta_{a}\right)-*\left(* \operatorname{dln} \beta \wedge \theta_{a}\right) \wedge * d \ln \beta\right)\right]=0,
\end{aligned}
$$

and the $g$-equation reads:

$$
\begin{aligned}
& 2 f_{0}\left[\left(\frac{1}{4} g^{a b} \mathcal{R}_{d}^{c} \wedge \eta_{c}^{d}+\frac{1}{2} \theta^{(a} \wedge \theta^{|c|} \wedge * \mathcal{R}_{c}^{b)}\right)-\right. \\
& +\rho_{1}\left[\mathcal{T}^{(a} \wedge * \mathcal{T}^{b)}+\frac{1}{2} g^{a b} \mathcal{T}_{c} \wedge * \mathcal{T}^{c}+\theta^{(a} *\left(* \mathcal{T}^{|c|} \wedge \theta^{b)}\right) \wedge * \mathcal{T}_{c}\right]+ \\
& +\rho_{2}\left(2 \delta_{d}^{(a} \mathcal{T}^{|c|} \wedge \theta^{b)}+\frac{1}{2} g^{a b} \mathcal{T}^{c} \wedge \theta_{d}-+\rho_{3}\left(2 \mathcal{T}^{(a)} \wedge \theta^{b)}+\frac{1}{2} g^{a b} \mathcal{T}^{c} \wedge \theta_{c}-\right.\right. \\
& \left.\left.-\theta^{(a} \wedge *\left(*\left(\mathcal{T}^{|c|} \wedge \theta_{c}\right) \wedge \theta^{b)}\right)\right) \wedge *\left(\mathcal{T}^{d} \wedge \theta_{d}\right)\right)+ \\
& +\xi\left(2 g^{a b} \mathrm{D} * \mathcal{Q}+\frac{1}{2} g^{a b} \mathcal{Q} \wedge * \mathcal{Q}-\theta^{(a} \wedge *\left(* \mathcal{Q} \wedge \theta^{b)}\right) * \mathcal{Q}\right)+ \\
& +\zeta\left(g^{a b} \mathcal{T}^{c} \wedge * \mathcal{T}_{c}-g^{a b} \theta^{c} \wedge \mathrm{D} * \mathcal{T}_{c}+\mathcal{T}^{(a} \wedge *\left(\mathcal{Q} \wedge \theta^{b)}\right)+\right. \\
& \left.+\frac{1}{2} g^{a b} \mathcal{Q} \wedge \theta^{c} \wedge * \mathcal{T}_{c}+\theta^{(a} \wedge *\left(* \mathcal{T}_{|c|} \wedge \theta^{b)}\right) \wedge *\left(\mathcal{Q} \wedge \theta^{c}\right)\right)+ \\
& \left.+l\left(\frac{1}{2} g^{a b} \mathrm{~d} \ln \beta \wedge * \mathrm{~d} \ln \beta-\theta^{(a} \wedge *\left(* \mathrm{~d} \ln \beta \wedge \theta^{b)}\right) \wedge * \mathrm{~d} \ln \beta\right)\right]- \\
& -\mathrm{D} \Lambda^{a b}-\frac{1}{4} \Lambda^{a b} \wedge \mathcal{Q}+\lambda^{a b}=0 .
\end{aligned}
$$

The variation of (2.2) with respect to $\beta$ gives the equation

$$
l \mathrm{~d} * \mathrm{~d} \ln \beta-\lambda^{a b} g_{a b}=0 .
$$


The variation (2.2) with respect to $\Lambda^{a b}$ gives the Weyl condition (1.1). It means, what spacetime has geometrical structure of a Cartan-Weyl space $C W_{4}$. The variation (2.2) with respect to $\lambda^{a b}$ gives the structure of the tangent space metric (1.2), which is realized at the certain most convenient choice of basis in each spacetime point. A consequence of (1.2) is representation of the Weyl 1-form as [12]

$$
\mathrm{Q}=q \mathrm{~d} \ln \beta, \quad q=-8 .
$$

After symmetrization of the equation (2.4) Lagrange uncertain multipliers $\Lambda^{a b}$ are determined and should be substituted in the equation (2.5) that allows then to determine Lagrange uncertain multipliers $\lambda^{a b}$. Representations (1.1) and (1.2) together with (2.7) should be substituted in the variational field equations (2.3)-(2.5), what results a manifestation in these equations of interaction of gravitational field with the scalar field $\beta$ in a Cartan-Weyl space $C W_{4}$.

After antisymmetrization of the equation (2.4) we receive the consequence of this equation, which is not containing the Lagrange uncertain multipliers,

$$
\begin{aligned}
& -\frac{1}{2} \mathcal{T}_{c} \wedge \eta_{a b}{ }^{c}+\eta_{a b} \wedge \mathrm{d} \ln \beta+2 \rho_{1} \theta_{[a} \wedge * \mathcal{T}_{b]}+2 \rho_{2} \theta_{[a} \wedge \theta_{|c|} \wedge *\left(\mathcal{T}^{c} \wedge \theta_{b]}\right)+ \\
& +2 \rho_{a} \theta_{a} \wedge \theta_{b} \wedge *\left(I^{a} \wedge \theta_{c}\right)+\zeta \theta_{[a} \wedge *\left(\mathcal{Q} \wedge \theta_{b]}\right)=0 .
\end{aligned}
$$

After contracting of the equation (2.8) externally on the right with $\theta^{a}$ and then applying Hodge dual operation, we shall receive the following consequence,

$$
\frac{2}{3}\left(1-\rho_{1}+2 \rho_{2}\right) \mathcal{T}=\left(\frac{1}{4}+\zeta\right) \mathcal{Q}
$$

where $\mathcal{T}={ }^{*}\left(\theta_{c} \wedge{ }^{*} \mathcal{T}^{c}\right)$ is a torsion trace 1 -form.

It is possible to conclude on the basis of (2.7) and (2.9) that as a consequence of the gravitational field equations the torsion trace 1-form can be represent as 


$$
\mathcal{T}=s d \ln \beta, s=\frac{3(1+4 \zeta)}{-1+\rho_{1}-2 \rho_{2}}=\text { const }
$$

After contracting of the equation (2.3) on the indexes $a, b$, one can obtain another consequence, which does not contain Lagrange uncertain multipliers,

$$
2\left(\rho_{1}-2 \rho_{2}+8 \zeta\right) \mathcal{T}+(16 \xi+3 \zeta) \mathcal{Q}=0
$$

3. Spherically symmetric solution for the central body in Cartan-Weyl space with WeylDirac scalar field

We shall search a solution in a spherically symmetric case in the form,

$$
d s^{2}=e^{-\mu} d t^{2}-e^{\mu}\left(d r^{2}+r^{2}\left(d \theta^{2}+\sin ^{2} \theta d \varphi^{2}\right)\right)
$$

where $\mu=\mu(r)$ [18]. Owing to spherical symmetry the torsion 2-form determines only by its trace that in the formalism of external forms reads, $\mathrm{T}^{a}=(1 / 3) \mathrm{T} \wedge \theta^{a}$. With the account of (2.7) and (2.10) the relation (2.9) will be written down as,

$$
2 s\left(1-\rho_{1}+2 \rho_{2}\right)+3 q\left(\zeta+\frac{1}{4}\right)=0 .
$$

As a result of calculation, one can convince that all components of the equation (2.8) for the metric (3.1) vanish as a consequence of the equality (3.2).

A calculation of the $\boldsymbol{\theta}$-equation with the account of (3.2) gives the following results. At $a=0$ we receive the equation 


$$
\begin{gathered}
2\left(\mu^{\prime \prime}+\frac{2}{r} \mu^{\prime}\right)+\left(\mu^{\prime}\right)^{2}=\left(\frac{1}{k}(\ln \beta)^{\prime}\right)^{2}, \\
k^{-2}=I+q\left(\xi+\frac{1}{2} q s \zeta+\frac{1}{8} q s-\frac{3}{64} q^{2}\right) .
\end{gathered}
$$

At $a=1, a=2$ and $a=3$ one obtains the identical equations

$$
\left(\mu^{\prime}\right)^{2}=\left(\frac{1}{k}(\ln \beta)^{\prime}\right)^{2} .
$$

Calculation of the trace of the $g$-equation results in the equation,

$$
\begin{aligned}
& \mu^{\prime \prime}+\frac{2}{r} \mu^{\prime}+\left(\mu^{\prime}\right)^{2}=\left(\frac{1}{k}(\ln \beta)^{\prime}\right)^{2}+ \\
& \left.+(\ln \beta)^{\prime \prime}+\frac{2}{r}(\ln \beta)^{\prime}\right)\left[-I+8\left(q \xi+\frac{1}{2} s \zeta+\frac{1}{8} s-\frac{3}{64} q\right)\right]
\end{aligned}
$$

Joint consideration of (3.2) and (2.11) gives a relation, 


$$
q \xi+\frac{1}{2}\left(\zeta+\frac{1}{4}\right) s-\frac{3}{64} q=0
$$

owing to which the field equations (3.3)-(3.6) become essentially simpler and reduce to the following three differential equations,

$$
\mu^{\prime \prime}+\frac{2}{r} \mu^{\prime}=0,(\ln \beta)^{\prime \prime}+\frac{2}{r}(\ln \beta)^{\prime}=0, \mu^{\prime}= \pm \sqrt{I}(\ln \beta)^{\prime} .
$$

which solution for the metric (3.1) and the Weyl-Dirac scalar field reads,

$$
\mu(r)=\frac{r_{0}}{r}, \quad \beta(r)=\beta_{\infty} \exp \left( \pm \frac{r_{0}}{\sqrt{l} r}\right)
$$

where $r_{0}$ and $\beta_{\infty}$ are an arbitrary constants of integration, where the value of $\beta_{\infty}$ describes the cosmological background of the Weyl-Dirac scalar field on the infinity determined by the value of the cosmological constant [5]-[11].

As a result a Cartan-Weyl space $C W_{4}$ appears with the metric

$$
d s^{2}=e^{-\frac{r_{0}}{r}} d t^{2}-e^{\frac{r_{0}}{r}}\left(d r^{2}+r^{2}\left(d \theta^{2}+\sin ^{2} \theta d \varphi^{2}\right)\right)
$$

This metric at the large $r$ will give the same experimental results, as well as the Schwartzshild metrics, if the constant of integration choose equal to the gravitational radius, 
$r_{0}=r_{g}=2 G m / c^{2}$. The metric (3.10) is known as Papapetrou-Yilmaz-Rosen (PYR) metric [19]-[21]. Interest to this metric [22] - [24] arises in connection with the fact that it does not contain a singularity on the gravitational radius.

According to (3.9) the density of the Weyl-Dirac scalar field grows inside of mass congestions that increases a gravitational field inside these congestions. In this connection in [8] the hypothesis that the Weyl-Dirac scalar field represents alongside with "dark energy" also the basic component of "dark matter" is stated.

The results were received within the framework of performance of the state task № 3.1968.2014/K of the Ministry of education and science of Russian Federation.

\section{References}

1. Babourova O. V., Frolov B. N., Zhukovsky V. Ch. (2006). Phys. Rev. D., V. 74., 064012-1-12.

2. Baburova O.V., Zhukovskiy V.C., Frolov B.N. (2008). Teoret. Math. Phys., T. 157, № 1, 64-78.

3. Babourova O. V., Frolov B. N., Zhukovsky V. Ch. (2009). Gravit. Cosmol., V. 15, №. 1,13-15.

4. Frolov B.N. (2003). Poincare gauge gravitational theory, MPGU, Moscow

5. Baburova O. V., Frolov B. N., Kostkin R. S. (2010) Dirac's scalar field as dark energy within the frameworks of conformal theory of gravitation in Weyl-Cartan space. ArXive, 1006.4761v2.

6. Baburova O. V., Frolov B. N., Kostkin R. S. (2011). Dirac's scalar field as an effective component of the dark energy and an evolution of the cosmological "constant". ArXiv, $1102.2901 \mathrm{v} 1$.

7. Baburova O. V., Kostkin R. S., Frolov B. N. (2011). Russian Physics Journal, V. 54, N 1, 121-123.

8. Baburova O.V., Frolov B. N. (2012). Mathematical problems of the modern theory of gravitation, Moscow: MPGU, Publishing house "Prometej".

9. Babourova O. V., Frolov B. N., Lipkin K. N. (2012). Gravit. Cosm., V. 18, № 4, 225-231.

10. Babourova O. V., Lipkin K. N., Frolov B. N. (2012). Russian Physics Journal, V. 55, 7, 855-857.

11. Babourova O. V., Frolov B. N. (2015). Physics Research International, V. 2015, Article ID 952181, 1-6.

12. Baburova O. V., Frolov B. N., Febres E. V. (2015). Russian Physics Journal, V. 58, N 2, $283-285$.

13. Babourova O. V., Lipkin K. N., Frolov B. N., Shcherban' V. N. (2013). Russian Physics Journal, V. 56, N 6, 722-724. 
14. Dirac P. A. M. (1973). Proc. Roy. Soc. A., V. 333, 403-418.

15. Deser S. (1970). Ann. Phys. (USA), V. 59, No 1, 248-253.

16. Weyl H. (1918). Raum, Zeit, Materie. Berlin: Springer.

17. Babourova O. V., Frolov B. N., Klimova T. A. (1999). Class. Quantum Grav., V.16, 1-14.

18. Babourova O. V., Frolov B. N., Febres E. V. (2015). Russian Physics Journal, V. 57, N 9, 1297-1298.

19. Papapetrou A. (1954). Z. Phys., V. 139, 518-532.

20. Yilmaz H. (1958). Phys. Rev., V. 111, 1417-142.

21. Rosen N. (1973). Gen. Rel. Grav. J., V. 4, 435-447.

22. Wyman M. (1981). Phys. Rev. D., V. 24, 839-841.

23. Kaniel S., Itin Y. (1997). ArXive, qr-qc/9707008.

24. Muench U., Gronwald F., Hehl F. W. (1998). Gen. Rel. Grav., V. 30, 933-961. 